$$
\begin{aligned}
& F=\begin{array}{llll}
1.4822 & 084 & E=0.0000000 & \gamma t=\varphi
\end{array} \\
& \text { - . } 748 \quad 384 \cos \varphi \\
& +.0097868 \cos 2 \varphi \\
& -.0001881 \cos 3 \varphi \\
& -.0000160 \cos 4 \varphi \\
& E=\begin{aligned}
& 0.0000000 \\
+ & .3860502 \sin \varphi
\end{aligned} \\
& -.0214468 \sin 2 \varphi \\
& +.0016039 \sin 3 \varphi \\
& -.0001027 \sin 4 \varphi \\
& \text { - .3799 440 sin } \varphi \\
& +.0193785 \sin 2 \varphi \\
& -.0014450 \sin 3 \varphi \\
& +.0000435 \sin 4 \varphi \text {, }
\end{aligned}
$$

déduites des séries de $\xi$ et $\eta$, mais ayant subi par la transformation une perte d'exactitude, nous avons, M. Bertelsen et moi, trouvé, comme première approximation, par les doubles quadratures de $\frac{\mathrm{d}^{2} E}{\mathrm{~d} \psi^{2}}$ et de $\frac{\mathrm{d}^{2} F}{\mathrm{~d} \psi^{2}}$ :

$$
\begin{aligned}
F= & 1.4822084 \\
& -.1748352 \cos x \psi \\
+ & .0097878 \cos 2 x \psi \\
& -.0001880 \cos 3 x \psi \\
+ & .0000166 \cos 4 x \psi \\
+ & .0000104 \cos 5 x \psi \\
& -.0000013 \cos 6 x \psi \\
+ & .0000001 \cos 7 x \psi
\end{aligned}
$$

$$
\begin{aligned}
E= & 0.0000000 \\
& +.3860502 \sin x \psi \\
& -.0214456 \sin 2 x \psi \\
& +.0016043 \sin 3 x \psi \\
& -.0001029 \sin 4 x \psi \\
& +.0000054 \sin 5 x \psi \\
& -.0000001 \sin 6 x \psi
\end{aligned}
$$

$$
\begin{aligned}
\gamma t= & x \psi \\
& -.3799402 \sin x \psi \\
& +.0193806 \sin 2 x \psi \\
& -.001444 \mathrm{sin} 3 x \psi \\
& +.0000448 \sin 4 x \psi \\
& +.0000028 \sin 5 x \psi \\
& -.0000009 \sin 6 x \psi \\
& +.0000001 \sin 7 x \psi
\end{aligned}
$$

ou $x=-6.056448 \quad \gamma=1.32166887$ (voir plus haut), pour la constante $K$ de l'intégrale de Jacobi j'ai trouvé

$$
K=\text { I } 3.190046 \text {, }
$$

quant à la constante $c$ employée par M. Burrau, on a

$$
c=-1 / 8 K=-1.648756 \text {. }
$$

Je pense que nos séries sont exactes dans leurs six premiers chiffres décimaux.

La convergence de ces séries est certainement supérieure à celle des $\xi$ et $\eta$ en fonction de $t$. Les queues en seront assez négligeables pour nous permettre d'attaquer des cas encore plus difficiles.

Avec les nouvelles coordonnées $E$ et $F$, nous n'avons pas redouté le cas limite des librations pures, celui de M. Burrau. Son orbite transformée doit étre représentée par une courbe ovale assez régulière, dont nous avons déjà une première approximation, et si nous préférons attendre des approximations ultérieures avant la publication de nos calculs, nous pouvons au moins annoncer que nons y avons retrouvé des queues assez embarrassantes dont les termes décroissent seulement à peu près dans le rapport de 5 à 2 .

Copenhague I 895 Février.

T. N. Thiele.

\title{
Nouvelle méthode de détermination des vitesses radiales des étoiles.
}

\section{Par Artémie Orbinsky.}

Les vitesses radiales des astres en général se déterminaient jusqu'à présent par trois méthodes: $\mathrm{r}^{\circ}$ celle de mesure directe des distances entre les raies spectrales de l'astre en question et les raies correspondantes d'un spectre artificiel, par exemple de l'hydrogène (M. Huggins et autres); $2^{\circ}$ celle de M. Vogel (Publicationen des Astrophysikalischen Observatoriums zu Potsdam, VII. Bd., r. Th., I. Messmethode); $3^{\circ}$ enfin M. Dunér dans ses $\gg$ Recherches sur la rotation du Soleil " mesurait les distances des raies spectrales du Soleil des raies telluriques voisines. M. E. C. Pickering voulait faire usage de cette dernière méthode pour mesurer les vitesses radiales d'après les spectrogrammes obtenus à l'aide de la méthode Fraunhofer (prisme devant l'objectif du télescope); il croyait encore profiter pour cela des raies d'absorption de quelques substances mises devant le prisme (Henry Draper Memorial, First Annual Report, Cambridge, Mass., 1887 ).

Je veux proposer ici encore la méthode suivante:

Soit $V$ la vitesse de la lumière, $v$ la vitesse radiale de l'astre; par le principe de Doppler-Fizeau on aura $\Delta \lambda=\lambda \frac{v}{V}$, où $\Delta \lambda$ est la variation de la longueur d'onde $\lambda$ due à la vitesse radiale $v$. En désignant par $n$ le nombre de révolutions du micromètre mesurant dans un $\mu \mu$ on aura en ces unités pour deux longueurs d'onde $\lambda_{1}$ et $\lambda_{2}$ les expressions $\mathrm{d} \lambda_{1}=n_{1} \Delta \lambda_{1}=n_{1} \lambda_{1} \frac{v}{V}, \quad \mathrm{~d} \lambda_{2}=n_{2} \lambda_{2} \frac{v}{V}$, d'où

$$
v=V \frac{\mathrm{d} \lambda_{1}-\mathrm{d} \lambda_{2}}{n_{1} \lambda_{1}-n_{2} \lambda_{2}} \text {. }
$$

Telle sera la vitesse absolue de l'étoile, si les distances $\mathrm{d} \lambda_{1}$ et $\mathrm{d} \lambda_{2}$ sont mesurées relativement aux raies spectrales d'une source de lumière immobile.

Si l'on photographie (soit à l'aide du spectrographe de M. Vogel, soit au moyen du prisme devant l'objectif) deux spectres côté-à-côté sur la même plaque, on pourra mesurer les quantités $\mathrm{d} \lambda_{1}$ et $\mathrm{d} \lambda_{2}$ lesquelles seront prises pour les raies d'un des spectres relativement aux raies correspondantes de l'autre. Ces mesures étant différentielles elles s'exécuteront avec une grande exactitude comme l'a démontré $M$. Vogel. Les constantes du spectrographe étant connues la formule (A) donnera la vitesse d'une source de lumière (astre étudié) relativement à celle de l'autre (de repère). Naturellement les longueurs d'onde $\lambda_{1}$ et $\lambda_{2}$ doivent être corrigées pour la vitesse de l'étoile de repère, supposée connue. Cette correction sera d'ailleurs négligeable dans la plupart des cas. 
La formule (A) montre qu' on pourra obtenir des résultats d'autant plus précis que la différence $n_{1} \lambda_{1}-n_{2} \lambda_{2}$ sera plus grande. Il est donc avantageux $\mathrm{I}^{\circ}$ que les limites des spectres photographiés soient aussi larges que possible, $2^{\circ}$ que le prisme (ou le système des prismes) employé donne une différence aussi grande que possible entre les dispersions des deux extrémités des spectres. Dans les conditions ordinaires des spectres prismatiques ces deux conditions sont contraires l'une à l'autre, mais la seconde est de beaucoup plus grave que la première. On voit aussi que les spectres prismatiques donnant une grande différence entre les dis. persions des diverses parties du spectre, sont plus avantageux que les spectres de diffraction.

En supposant qu' on ait des spectres prismatiques et $\lambda_{1}<\lambda_{2}$ on aura toujours $n_{1} \lambda_{1}-n_{2} \lambda_{2}>0$ et le signe de la vitesse $v$ dépendra du signe de $\mathrm{d} \lambda_{1}-\mathrm{d} \lambda_{2}$. Pour suffire à la convention existante sur le signe de $v$ on devra compter $\mathrm{d} \lambda>0$, si la raie du spectre étudié est déplacée vers le bout moins refracté du spectre relativement à la même raie du spectre de repère. On peut exprimer cela aussi de la manière suivante: l'étoile étudiée s'éloigne ou s'approche de l'observateur en comparaison avec l'étoile de repère suivant que son spectre est plus court ou plus long que celui de l'étoile de repère entre les mêmes raies.

Pour voir l'ordre des grandeurs $\mathrm{d} \lambda_{1}-\mathrm{d} \lambda_{2}$ je donne les exemples suivants: $I^{\circ}$ dans les conditions $d u$ spectrographe à deux prismes de $\mathrm{M}$. Vogel une vitesse radiale de $10 \mathrm{~km}$ correspond à $\mathrm{d} \lambda_{1}-\mathrm{d} \lambda_{2}=0.0085 \mathrm{~mm}$, si l'on prend le spectre entre $468 \mu \mu$ et 4 I $\circ \mu \mu$, pendant que le déplacement de la raie $H \gamma$ pour la même vitesse est $0.0125 \mathrm{~mm} ; 2^{\circ}$ dans les conditions auxquelles on obtient les spectres de la plus grande dispersion à Harvard College Observatory (prisme de $20^{\circ}$ d'angle réfringent devant l'objectif du Draper télescope) la même vitesse radiale de $10 \mathrm{~km}$ correspond à $\mathrm{d} \lambda_{1}-\mathrm{d} \lambda_{2}=0.0320 \mathrm{~mm}$ si l'on prend le spectre entre $489 \mu \mu$ et $395 \mu \mu$.

Tout ce qui a été dit plus haut suppose une invariabilité de la position du prisme (ou des prismes) relativement aux rayons de la lumière analysée de l'une et de l'autre source. Dans le cas du spectrographe de M. Vogel cette invariabilité est bien assurée. C'est une autre affaire pour le spectrographe de Fraunhofer. Les remarques suivantes auront lieu dans ce dernier cas : les changements éprouvés par le prisme relativement à l'objectif peuvent être divisés comme il suit, $1^{\circ}$ ceux qui troublent le parallélisme de deux spectres (rotation du prisme entre deux poses autour de l'axe optique du télescope); cette correction sera toujours à rejeter, $2^{\circ}$ ceux qui déplacent l'un des spectres le long de l'autre ne troublant pas leur parallélisme (changements de position du prisme relativement à l'axe optique de l'objectif principalement). Que l'on fasse l'arête réfringente du prisme parallele au mouvement diurne des étoiles, ce dernier déplacement se confondra avec le déplacement produit par la différence des fautes du pointage du télescope en déclinaison pour les deux étoiles. Relativement à la deuxième faute beaucoup plus complexe et considérable on peut remarquer ce qui suit: si $i, r, r^{\prime}, i^{\prime}$ sont les angles faits par le rayon de la lumière analysée consécutivement avec les deux plans réfringents du prisme on aura

$$
\frac{\mathrm{d} i}{\mathrm{~d} i^{\prime}}=-\frac{\cos r \cos i^{\prime}}{\cos r^{\prime} \cos i} .
$$

Si le prisme se trouve à peu près au minimum de déviation d'une raie déterminée, $H \gamma$ par exemple, on aura pour cette raie à peu près $\mathrm{d} i=-\mathrm{d} i$. L'échelle angulaire des plaques (longueur focale de l'objectif) étant connue on pourra mesurer la distance angulaire entre les raies de la même longueur d'onde (et nommément celle pour laquelle le prisme se trouve au minimum de déviation) dans les deux. spectres ce qui donnera $\mathrm{d} i$ et par conséquent $\mathrm{d} i$. Comme dans la distance angulaire entre l'effet des vitesses radiales de deux étoiles, la question pourra être résolue rigoureusement par des approximations successives. Avec la quantité $\mathrm{d} i$ on calculera par la formule (B) les variations correspondantes $\mathrm{d} i_{1}$ et $\mathrm{d} i_{2}$ des raies au moyen desquelles on détermine la vitesse radiale. En transformant ces quantités angulaires en quantités linéaires (on les multipliera par la longueur focale de l'objectif et par la sécante de la distance angulaire de ces raies de la raie, $H \gamma$ par exemple, pour laquelle le prisme se trouve au minimum de déviation) on prendra leur différence et avec cette différence on corrigera la quantité $\mathrm{d} \lambda_{1}-\mathrm{d} \lambda_{2}$ mesurée.

On peut espérer toutefois que tous ces déplacements du prisme sont faciles à annuler et dans ce cas la formule (A) suffira seule à tous les calculs.

Je ferai encore quelques remarques sur la méthode proposée : $\mathrm{I}^{\circ}$ les avantages de la méthode de $\mathrm{M}$. Vogel, consistant en ce qu' on y mesure une quantité de raies au lieu d'une seule comme dans la méthode de mesures directes, sont pleinement conservés: on peut profiter d'une quantité de raies à chaque bout du spectre étudié; $2^{\circ}$ la méthode proposée dispense des spectres artificiels, c. ̀̀. d. d'un appareil assez complexe; $3^{\circ}$ elle introduit dans ce champ de mesures les clichés obtenus à l'aide d'un prisme devant

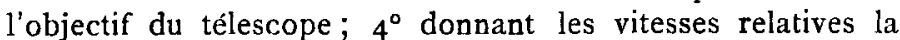
méthode proposée exige la connaissance des vitesses radiales absolues d'un certain nombre d'étoiles (étoiles de repère); ces étoiles fondamentales doivent être choisies parmi les plus brillantes comme donnant les spectres le plus vite possible et admettant la plus grande exactitude possible de détermination des vitesses radiales (grâce à l'admissibilité des plus grandes dispersions). Cette partie du problème se résout à l'aide du spectrographe de $\mathrm{M}$. Vogel. On pourrait encore profiter pour cela des vitesses mesurées avec des instruments plus puissants comme celui de l'Observatoire de Lick par exemple.

Ayant déterminé les vitesses absolues d'un nombre suffisant des étoiles de repère on choisira toujours entre elles celle qui donnera les conditions les plus favorables pour être photographiée avec l'étoile étudiée à un moment donné et dont le spectre soit le plus semblable au spectre de la dernière. Pour plus d'exactitude enfin et pour avoir possibilité d'exclure les changements progressifs des appareils pendant la pose on prendra deux photographies du spectre de l'étoile de repère - avant et après la pose de l'étoile étudiée. 